\title{
An Integrated Approach of Analytical Chemistry
}

\author{
Miguel de la Guardia
}

\author{
Department of Analytical Chemistry, University of Valencia, \\ 50 Dr Moliner St 46100 Burjassot - Valencia, Spain
}

\begin{abstract}
O desenvolvimento impressionante de métodos físicos de análise oferece um número expressivo de ferramentas para determinar, simultaneamente, um grande número de elementos e compostos a um nível de concentração muito baixos. A Química Analítica de hoje fornece meios apropriados para resolver problemas técnicos e obter informações corretas sobre sistemas químicos, de maneira a orientar técnicos e as decisões mais adequadas para a solução de problemas.

Nos anos recentes, o desenvolvimento de novas estratégias de amostragem, tratamento de amostras e exploração dos dados, através de pesquisas em amostragens de campo, procedimentos por microondas e quimiometria, em adição à revolução da metodologia analítica proveniente do desenvolvimento dos conceitos de análise em fluxo e análises de processos, oferece uma ligação entre a moderna instrumentação e problemas sociais ou tecnológicos.

A abordagem integrada da Química Analítica significa a necessidade de incorporar corretamente os desenvolvimentos em todos os campos da química básica, instrumentação e teoria da informação, em um esquema que considere todos os aspectos da obtenção e interpretação dos dados, levando ainda em consideração os efeitos paralelos das medidas químicas.

Neste artigo, novas idéias e ferramentas para análise de traços, especiação, análise de superfícies, aquisição e tratamento de dados, automação e descontaminação são apresentadas em um contexto da Química Analítica como uma estratégia de solução de problemas, focalizada na composição química de sistemas e aspectos de mérito específicos das medições analíticas, como exatidão, precisão, sensibilidade, seletividade, mas também velocidade e custos.

Os problemas tecnológicos e industriais, assim como os ambientais, os da medicina e os sociais, tem sido considerados como desafios cuja solução pelos químicos envolve a seleção das ferramentas mais apropriadase o desenvolvimento de estratégias adequadas.
\end{abstract}

The tremendous development of physical methods of analysis offers an impressive number of tools to simultaneously determine a large number of elements and compounds at very low concentration levels. Todays Analytical Chemistry provides appropriate media to solve technical problems and to obtain correct information about chemical systems in order to take the most appropriate decisions for problem solving.

In recent years the development of new strategies for sampling, sample treatment and data exploitation through the research on field sampling, microwaveassisted procedures and chemometrics, additionally the revolution of the analytical methodology provided by the development of flow analysis concepts and process analysis strategies offer a link between modern instrumentation and social or technological problems.

The integrated approach of Analytical Chemistry requires correctly incorporating the developments in all of the fields of both, basic chemistry, instrumentation and information theory, in a scheme which considers all aspects of data obtention and interpretation taking also into consideration the side effects of chemical measurements.

In this paper, new ideas and tools for trace analysis, speciation, surface analysis, data acquisition and data treatment, automation and decontamination, are presented in the frame of Analytical Chemistry as a problem solving strategy focused on the chemical composition of systems and on the specific figures of merit of the analytical measurements, like accuracy, precision, sensitivity, selectivity but also speed and cost.

Technological and industrial, but also environmental, health and social problems, have been considered as challenges for which solution the chemist should select the most appropriate tool and to develop an appropriate strategy.

Keywords: analytical chemistry, analytical strategies, traceability, flow analysis, clean methods

e-mail: miguel.delaguardia@uv.es 


\section{Some Approaches for a Definition of Analytical Chemistry.}

Nowadays, there are several definitions of Analytical Chemistry through the literature and probably the largest effort on this aspect has been carried out by the Working Party of the Federation of the European of Analytical Chemistry Societies (FECS) through the project of the development of an eurocurriculum on this matter ${ }^{1}$ and the meeting organized by Prof. Malissa and the Fresenius Journal in 1992 to establish an appropriate definition of today's analytical chemistry ${ }^{2}$.

The fact that a definition is not only a sentence, but it has a tremendous importance on the development and teaching of a discipline, can be clearly appreciated by looking at the tentatives carried out by K. Caumann, M. Valcárcel, A.M. Zuckerman and Zhon Nan, who tried to establish the limits and main characteristics of Analytical Chemistry in very long sentences which, in my opinion, try to define this discipline in the frame of Chemistry and nature sciences, to evidence the metrological characteristics of the analytical process and to insist on the different aspects from which Analytical Chemistry operates as a tool to obtain information and solving problems.

In the context of the $22^{\text {nd }}$ Annual Meeting of the Sociedade Brasileira de Química, I would like to offer to my colleagues of different disciplines in Chemistry a modern and integrated approach of today's Analytical Chemistry, which could be understood not as a definition but as a proposal for making work the analytical sense and to incorporate the modern developments in some different fields, such as those on automation, chemometrics, separation and measurement techniques, and sampling and sample pretreatment, in the frame of the analytical process as a unit which should integrate all these different aspects in order to catch an objective based on the knowledge of the composition and structure of the matter, but taking also into consideration the side effects of the process.

Basically, I think that Analytical Chemistry is more a point of view on the matter than a part or a subdiscipline of Chemistry. Obviously, Analytical Chemistry is Chemistry but not at all a part. It is all the Chemistry, from inorganic to organic, taking also into consideration biochemical aspects.

However, as depicted in Fig. 1, Analytical Chemistry is the Chemistry of the differences. From an analytical point of view, analogies of elements, of a same column or period of the periodic table, are left aside in the same way that analogies of organic compounds, having the same functional groups, in order to pay more attention to the specific identity of elements and compounds. In this sense, Analytical Chemistry is closer to the evidences of the life experiences than other chemical disciplines and it can be well understood by our students, who appreciate the different

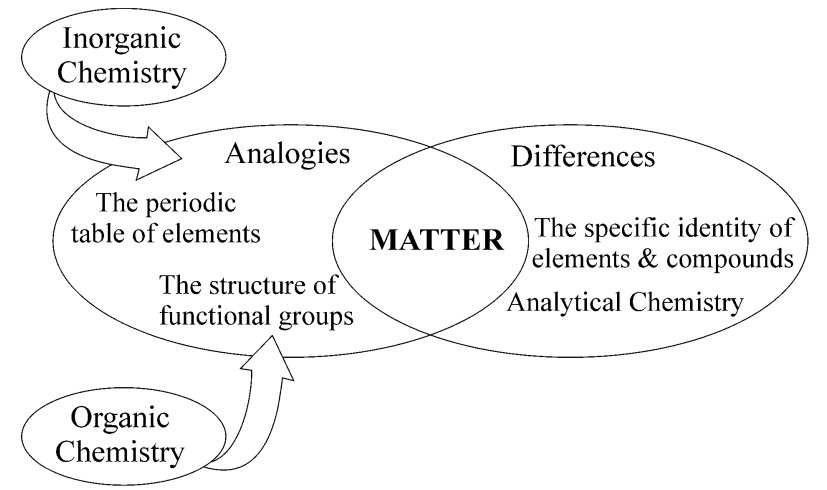

Figure 1. Analytical Chemistry as the Chemistry of the differences.

effects of sodium and potassium on soil fertility, in spite of the fact that both are alkaline elements, or the tremendous differences between the toxicity of methanol and ethanol, which have the small difference of a carbon and two hydrogen atoms.

So, it can be concluded that Analytical Chemistry is not at all a part of Chemistry but all the Chemistry seen from a particular view point, which pays more importance to the identity details of the different atoms, molecules or arrangements of both than to their similarities.

On the other hand, there are strong difficulties to differentiate Analytical Chemistry, Inorganic Chemistry or Organic Chemistry from Physicochemistry, because at the end it is true that this latter discipline provides the basis of the interpretation of all the phenomena related to the chemical structure of the matter.

However in the case of Inorganic, Organic or Biochemistry, the differences result from the reduction of the field considered by these disciplines, which provide a more in deep look than that provided by Physicochemistry.

In the case of Analytical Chemistry it can be appreciated that any analytical method is integrated by both, a theoretical background and a practical experimentation, and, from this perspective it can be said, as Prof. Kolthoff, that "Theory guides but experiment decides". So, Analytical Chemistry should be considered as the Chemistry of the experiments and, in this perspective, the research on Analytical Chemistry must provide not only new evidence or new methods of analysis, but methods which clearly improve the main features of previous ones. In this way the literature on Analytical Chemistry must provide new and original approaches, but also a clear validation of the suitability of those to solve analytical problems and to analyze natural samples.

When we consider Analytical Chemistry as a tool for problem solving it is necessary to clearly establish the main aspects of the discipline, the milestones which support the analytical work and provide its specific sense. 
As is indicated in Fig. 2, there are two main aspects to Analytical Chemistry: i) traceability and ii) the analytical properties.

Traceability is a property of samples and analytical methods, which refers to the production or behaviour, in fact to the story of the sample or the description, step by step, of the method and it especially describes the relationship of data with standards and has a tremendous importance for establishing the accuracy of analytical measurements of one or several laboratories in their quality assurance and harmonization ${ }^{3}$.

In a practical sense traceability can be considered as the careful control of all steps concerning the analytical process, from sampling to results and so, it involves physicochemical, experimental and chemometric aspects as well as than practical aspects concerning a representative and unbiased sampling and a clear identification and control of the samples through the whole process.

On the other hand, the analytical properties are the main support of the analytical viewpoint. Accuracy, representativity, precision, selectivity and sensitivity are the main characteristics of a method, which are considered when the analyst is searching for the most appropriate methodology in order to obtain chemical information from a system and, in this sense, analytical properties should be permanently considered as the guide for sample analysis and comparison between analytical strategies and methods. However, in real work, there are not only basic principles, which should be taken into consideration, but also some practical aspects as the time required to perform sample analysis, the cost of analysis, safety and comfort considerations for making analysis and side-effects, this being an important environmental consideration.

Valcárcel and $\operatorname{Rios}^{4,5}$ have evaluated the hyerarchy of the analytical properties and established the relationships between all these. In the following sections we will describe an integrated approach of Analytical Chemistry, which

\section{Analytical Chemistry}

Traceability The careful control of all the steps concerning the analytical process from sampling to results

Analytical Properties

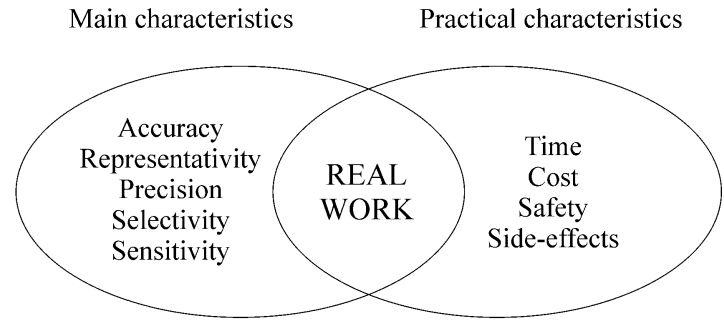

Figure 2. Main aspects of Analytical Chemistry. could consider simultaneously all the basic and practical aspects of the analysis.

Nowadays, it is very frequent to say that the problem and not the sample is the focus of analysis ${ }^{6,7}$ trying to integrate both aspects, the problem solving strategy and the analytical features considered. Figure 3 depicts a single scheme to orientate the analytical work considering the different steps which lie problem, sample, analytical method and results and, as it can be seen in this figure, samples must be representative, all the steps of the analytical method must be carefully verified and the traceability must be permanently controlled and checked in order to obtain appropriate solutions or recommendations for problem solving.

When we consider the resolution of problems in a real world context, it is necessary to think about the main restrictions imposed by the time required to provide data for solving important or hazardous problems in a limited period of time. In this sense, Analytical Chemistry has a double and contradictory objective: to provide the maximum possible information at the minimum cost.

As indicated in Fig. 4 a quantitative, or at least semiquantitative information about the concentrations of as many as possible elemental components and compounds could be necessary to solve an analytical problem, these

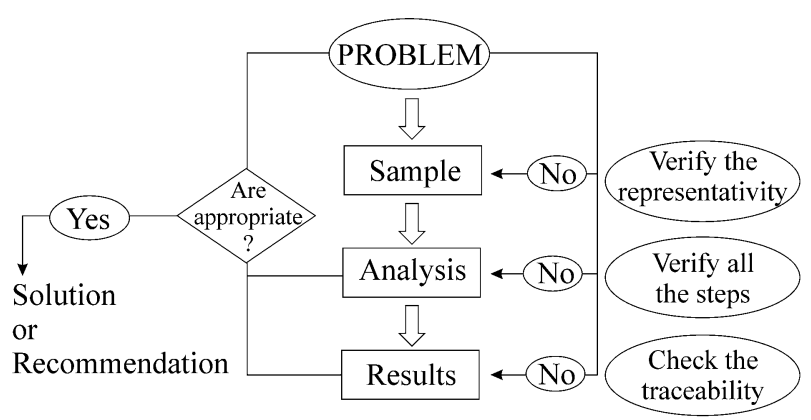

Figure 3. Analytical Chemistry: a tool for problem solving.

\section{MAXIMUM INFORMATION}

\begin{tabular}{|c|c|}
\hline $\begin{array}{l}\sum_{2} \text { Quantitative or at least } \\
\text { semiquantitative data }\end{array}$ & MINIMUM COST \\
\hline $\begin{array}{l}\sum_{2} \text { Simultaneous or fast sequential } \\
\text { multielemental analysis }\end{array}$ & $\begin{array}{l}\text { Direct analysis without } \\
\text { sample preparation }\end{array}$ \\
\hline $\begin{array}{l}\text { Ly Information about species } \\
\text { not only total content }\end{array}$ & $\begin{array}{l}\text { Safe \& confortable } \\
\text { conditions }\end{array}$ \\
\hline $\begin{array}{l}\text { Integration of analytical data } \\
\text { with other information }\end{array}$ & No-side effects \\
\hline $\begin{array}{l}\sum_{2} \text { Integration of thermodynamic } \\
\& \text { knetic data }\end{array}$ & $\begin{array}{l}\text { Low cost methods } \\
\text { Fast methods }\end{array}$ \\
\hline$\sum_{2}$ Real time monitoring & \\
\hline
\end{tabular}

Figure 4. Requirements of problem solving Analytical Chemistry. 
informations being simultaneously obtained, or at least in a fast sequential way. Information about species, and not only about total content of elements, is also required to establish the toxic or pollution level. Additionally, all chemical information, obtained from both thermodynamic and kinetic data, is required to be integrated with other climatological and social data. Finally, informations obtained needs, in some cases, to be monitored in real time. So, it is easy to imagine the large effort required to obtain a complete picture of data concerning a problem and the need for establishing the limits of that.

On the other hand, for practical reasons, the cost of analysis should be reduced in terms of time consumed, prize, safety and comfort conditions of operation and sideeffects of the analytical methodologies, to avoid environmental pollution and production of toxic wastes. Therefor direct monitoring methods involving the use of nontoxic reagents and suitable to be employed without sample preparation could be the best choice.

\section{Main Keywords of Today's Analytical Chemistry}

The main keywords, which any practitioner of Analytical Chemistry could identify in the scientific literature, concern: i) traceability, ii) chemometrics, iii) flow-analysis and robotics, iv) in-field sampling, v) microwave-assisted treatments, vi) hyphenation, vii) speciation viii) sensors, ix) screening methodologies and $\mathrm{x}$ ) decontamination of wastes. All these words come from the main problems and challenges of today's analytical work, such as the need for developing the identity of this discipline, the treatment of tremendous amounts of information, the automation of analytical processes, the improvement of sampling and sample preservation, the enhancement of slow thermal treatments required for sample preparation and analyte derivatization, the lack of highly selective methods suitable for the direct differentiation of organic molecules and their metabolites, or different species of a same element, and the need for sustainable strategies for environmental protection.

All the main keywords of today's Analytical Chemistry can be related with one or several steps of the analysis and appear as new frontiers for solving the real problems of today.

Figure 5 summarizes some of the problems found to obtain as much as possible correct information from samples in the different steps of the analysis, from sampling, sample storage and sample preservation to the determination itself, data treatment and waste decontamination. The main thesis of this work is that both, chemometrics and flow analysis, are the most relevant approaches suitable for the integration of all the aforementioned steps and the correct introduction of enhanced methodologies for problem solving.

\section{Goals for the Improvement of Analytical Methods}

In the past, the main efforts were focused on the development of new, more sophisticated methods of measurement based on physicochemical principles and thus, nowadays, there are a large number of different methodologies and techniques suitable for adapting intelligently to solve any specific problem. However, at this point, it is necessary to pay attention to other steps of the analytical process such as: i) sampling and sample storage, ii) sample pretreatment, iii) selection of the most appropriate technique, iv) hyphenation between analytical techniques, especially between separation and determination, v) data exploitation and vi) waste decontamination. All these questions can be appropriately integrated by using two concepts, that of flow analysis and that of chemometrics, and improved by incorporating recent developments in solid phase, supercritical fluid extraction, microwave-assisted treatments, electrophoresis and chromatography.

\begin{tabular}{|c|c|c|}
\hline Step & Problems & New Frontiers \\
\hline Sampling & $\begin{array}{l}\text { Representativity } \\
\text { Fast information requirement }\end{array}$ & $\begin{array}{l}\text { Chemometric models for sampling } \\
\text { In field sampling } \\
\text { Screening techniques }\end{array}$ \\
\hline Sample storage & $\begin{array}{l}\text { Lost or contamination of analytes } \\
\text { Modification of analyte environment }\end{array}$ & $\begin{array}{l}\text { On-line preconcentration through flow } \\
\text { treatment } \\
\text { Sensors }\end{array}$ \\
\hline Sample preparation & $\begin{array}{l}\text { Tedious \& time consuming extraction or } \\
\text { dissolution steps }\end{array}$ & $\begin{array}{l}\text { Direct ATR or X-Ray analysis of solids } \\
\text { Direct analysis through the use of slurries } \\
\text { Microwave-assisted digestion \& extraction }\end{array}$ \\
\hline Determination & $\begin{array}{l}\text { Sensitivity } \\
\text { Selectivity } \\
\text { Speciation capabilities }\end{array}$ & Preconcentration \\
\hline
\end{tabular}

Figure 5. Problems and new frontiers of the different steps of Analytical Chemistry. 


\section{Sampling and Sample Storage}

Regarding sampling and sample storage, it is well known that the quality of data strongly depends on the appropriate selection and representativity of samples and their correct identification, as well as on the changes of sample condition from its own environment to the laboratory, this being the true Achilles heel of any analytical method, independent of the use of the most sophisticated instrumentation.

As a general principle, samples must be analyzed as soon as possible and maintained in their own environment until the moment to be treated. Obviously in some cases this situation is just a desire and samples must be taken from far places, stored in containers, transported and analyzed only after several days. These are the main reasons for serious uncertainties about the identity between data obtained and true values of some of the parameters measured.

A practical problem, which clearly evidences the need for appropriate sampling and integrated measurement techniques, is the study of chemiclines in deep lakes, that is, the distribution of chemical species as a function of the depth of a lake and taken into account the presence or absence of light and oxygen, the pressure of dissolved gases, such as $\mathrm{CO}_{2}$, which strongly depends on the height of the water layer, and especially on the redox potential, which affects the stability and solubility of the different chemical species. As it can be seen in Fig. 6, use of the concepts of flow analysis makes possible the sampling at different depths and assures their transport and measurement without changing their environment and thus, redox potential, $\mathrm{pH}$, dissolved $\mathrm{O}_{2}$ and some free anions and cations, could be determined directly. On the other hand the use of well selected solid phases, can assure the on-line selective extraction and preconcentration of trace elements and organic compounds which can be retained in anionic or cationic exchange resins or $\mathrm{C}_{18}$ cartridges and thus, preconcentrated, fixed and stabilized also reducing the cost of sample transport and the risks of contamination.

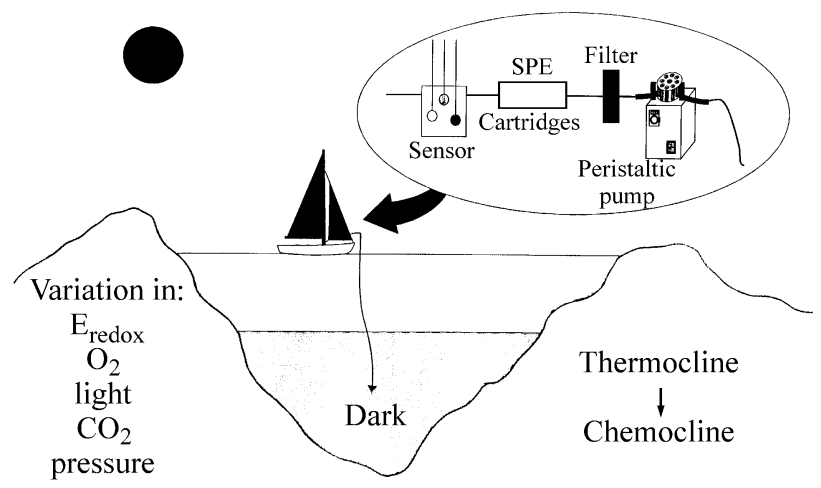

Figure 6. Scheme of a flow manifold for the in-situ study of lake chemistry at different deep levels.
So, flow analysis concepts ${ }^{8-10}$, which at the beginning were applied to the automation of the measurement process $^{11}$ offer today excellent possibilities for improvement of sampling and reducing the risks of sample storage.

On the other hand this is the time for the application to solve real problems through the use of non-destructive methodologies capable of being applied directly to the sample and in vivo techniques based on the technology of sensors, both chemical and biochemical, devices on which a tremendous research effort has been carried out in the last decade $^{12}$.

\section{Sample Pretreatment}

In the last 50 years, Analytical Chemistry has been dramatically changed by the incorporation and development of physical methods, which combine both, the recognition of chemical compounds and the measurement of electrical magnetic or optical properties. That has improved the limits of detection of classical methods of analysis and increased the possibilities of multicomponent determinations in a sample. However from the old past times the main techniques of sample preparation and especially those concerning sample dissolution remained unmodified and so, classical convective heating practices and dry-ashing or wet-ashing procedures continue to be employed.

Fortunately, in recent years the development of sonochemistry ${ }^{13}$ and the introduction of microwave-assisted procedures for sample digestion ${ }^{14}$ and extraction of pollutants ${ }^{15}$ have improved the sample pretreatment in the methods of analysis.

Figure 7 summarizes the energy sources commonly employed for sample solution and, as can be seen, the introduction of low energy sources, like ultrasound, provides a good way for avoiding changes in species stability, due to their thermal decomposition, and the introduction of microwave-assisted procedures involves the acceleration of thermal processes and thus, the simultaneous use of microwaves and acids and/or appropriate reagents opens new

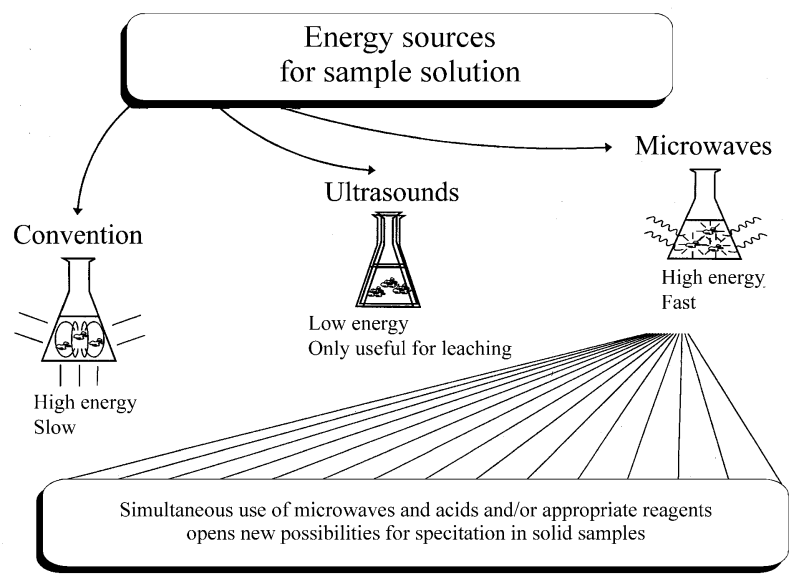

Figure 7. Energy sources employed for sample dissolution. 
possibilities for speciation and fast analysis of solid samples, and being also possible to integrate these processes on-line by reducing the pretreatment steps from several hours to a few minutes or seconds ${ }^{16-18}$. Additionally, electrochemical dissolution processes can solve some practical problems with a simple set-up and also avoid the use of chemical reagents.

\section{Selection of Appropriate Methods of Analysis}

A trip through the analytical literature reveals that it is possible to identify a lot of proposed procedures to carry out any determination and this inflaction of methods, which is in principle a parameter of the good health of our discipline, can cause terrible drawbacks to those who try to apply a method to solve a specific problem without having a lot of time to evaluate all the advantages and problems inherent to the different methodologies.

There are four main criteria to choose an analytical procedure: i) to evaluate the whole procedure, ii) to evaluate the main figures of merit of the different available procedures, iii) to adapt the methodologies to the available media and instrumentals and iv) to develop integrated strategies.

In fact to choose the most appropriate procedure to solve an analytical problem requires to think from the analytical point of view and to look for the improvement of analytical properties, as accuracy, precision, sensitivity and selectivity, but also thinking on speed, safety and comfort and especially cost and laboratory productivity.

An important recommendation to select the most appropriate methodology is to think of the whole process which is comparable to the development of an appropriate strategy for playing chess. To be a good player it is necessary to think not only step by step but to evaluate the initial steps, taking into consideration their effect on following ones.

However there is no single technique which can produce a complete picture of an analytical problem and because of that, an intelligent combination of informations obtained from several approaches is always the best choice.

\section{Hyphenation in Analytical Chemistry}

The sensitivity of instrumental procedures in some cases is not totally accompanied by a comparable selectivity and because of that, when complex samples are directly analyzed, the combination between separation procedures and, as sensitive as possible, detection it is necessary to assure both, selectivity and sensitivity of analytical determinations.

Hyphenation ${ }^{19}$ is a main keyword of today's Analytical Chemistry and it means the coupling between different techniques and instruments, in order to improve the determinations by creating synergistic combinations.
The on-line coupling between high performance liquid chromatography (HPLC) and atomic spectrometry or that between gas chromatography (GC) and Fourier transform infrared spectrometry (FTIR) or mass spectrometry (MS) are good examples of the potential for hyphenation processes. On the other hand, the correct integration of both, instruments and chemical processes, such as sample pretreatment and precolumn or postcolumn derivatization, are necessary to assure fast, selective and sensitive determinations and for that, once again, the use of flow analysis strategies is very convenient to perform all these steps in as short as possible time interval, thus avoiding contamination risks.

\section{Chemometrics}

Chemometrics $^{20,21}$ is a new discipline which refers to the statistical and computational treatment of chemical data and, in the last twenty years, has provided exciting new tools to improve experimental design and process optimization and to enhance the multivariate calibration strategies.

The capability of analytical techniques to produce information and the need of integrating not only physical and chemical data but also additional data to obtain a good picture of the elements involved in problem solving, requires the development and use of powerful calculation tools for data evaluation (such as data variance analysis and calibration strategies) and also for optimization of analytical variables and for correctly planning sampling and analysis.

The challenge, once again, is to be able to integrate on-line data treatment with data acquisition and, if possible, it could be convenient to fully automatize the analytical process, including corrective actions to be automatically taken. That involves a hard integration of all the steps of the analytical process in order to correctly ensure the quality of data and procedures and to enhance the process of making decisions without delay.

So, nowadays it is absolutely necessary to incorporate the modern multiparametric calibration tools and the complex data treatments, based on pattern recognition, networks and expert systems configuration, in order to explore in depth the information provided by the analytical instruments.

Figure 8 summarizes some of the main contributions of chemometrics to the improvement of Analytical Chemistry. 


\section{Chemometrics}

Adequacy of statistical models to the size of analytical data populations

Bayessian treatment of the accuracy problem

Planification of sampling as a function of variables which could affect data

Optimization of analytical parameters

Calibration, especially multivariate calibration

Integration of data obtained from different sources

Development of behavior models and prediction models

Full automation of measurement and control process

Figure 8. Chemometrics as an analytical tool.

\section{Environmentally Friendly Analytical Chemistry}

In past times, after data evaluation, it was considered that an analytical procedure was finished when all the elements to solve a problem were on the table. However, now-a-days, it is imposed by laws and by the compromise between scientists and our world to consider also the side effects of our job. In this new scenario, it is necessary to evaluate and to treat the wastes generated by the measurement processes in such a way that the less pollutant methods would be selected and the laboratory wastes decontamined $^{22}$

The environmental mentality in Analytical Chemistry is a recent compromise of chemistry but should be carefully considered in order to assure the sustainable development of our discipline.

Figure 9 summarizes the different stages in the evolution of the ecological mentality of the analytical laboratories, from the technical development to the bad conscience

Ecological mentality in the analytical laboratories

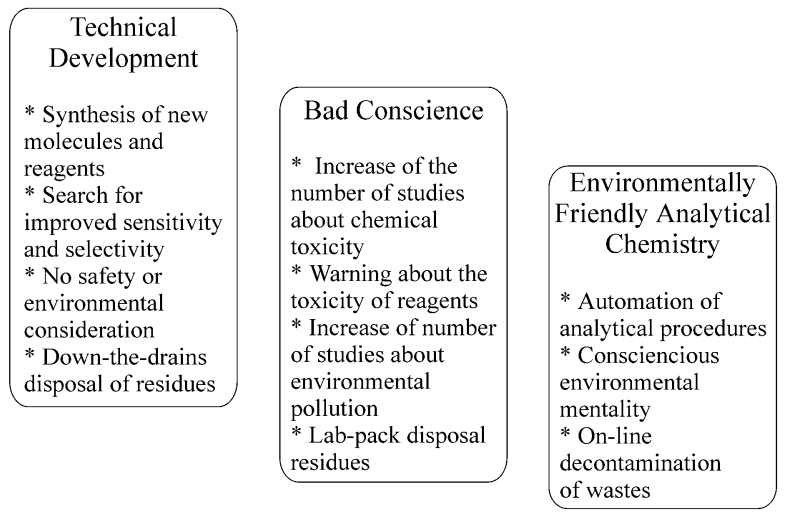

Figure 9. Stages of the evolution of ecological mentality in the analytical laboratories. of the environment damage and the engagement of sustainable practices.

The term "clean analytical chemistry" means a series of practices and strategies which reduce or avoid the pollutant side effects of the analytical methods. However, in order to maintain the main features of Analytical Chemistry without sacrificing important parameters, such as cost and speed of analysis, it is clear that the improvement of analytical methods should be focused on: i) reduction of wastes and miniaturization and ii) online decontamination or recycling of analytical wastes.

Once again, it is important to ensure that new developments on analytical methodologies could be integrated in the whole process and carried out without delay in sample treatment and data acquisition. As for example, Fig. 10 depicts two approaches on the aforementioned process. In one case, wastes of $\mathrm{mg} \mathrm{L}^{-1}$ of phenols determined by reaction with $50 \mathrm{mg} \mathrm{L}^{-1} \mathrm{p}$-aminophenol are on-line decomposed through a photo-assisted catalytic process ${ }^{23}$. In the another example, the $\mathrm{CCl}_{4}$, employed as carrier and solvent in the determination of ketoprofen by FTIR is on-line recovered by distillation ${ }^{24}$.

Nowadays, a series of thermal degradation, oxidation with $\mathrm{O}_{3}$ or $\mathrm{H}_{2} \mathrm{O}_{2}$, photodegradation and biodegradation strategies have been proposed for on-line decontamination of analytical wastes without requiring separate processes but on-line integration of the waste treatment to obtain finally a clean waste.

Concerning the reduction of reagent consumption, the use of flow techniques, from the pioneering work of Skeggs
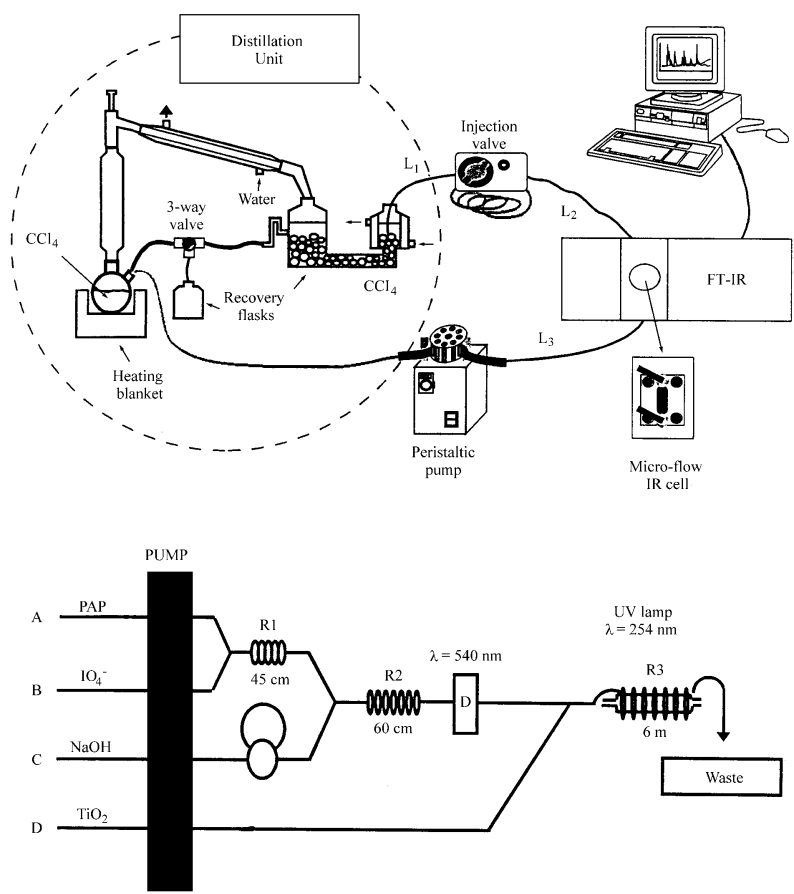

Figure 10. Manifolds employed for the development of clean analytical methods. 
Flow Analysis

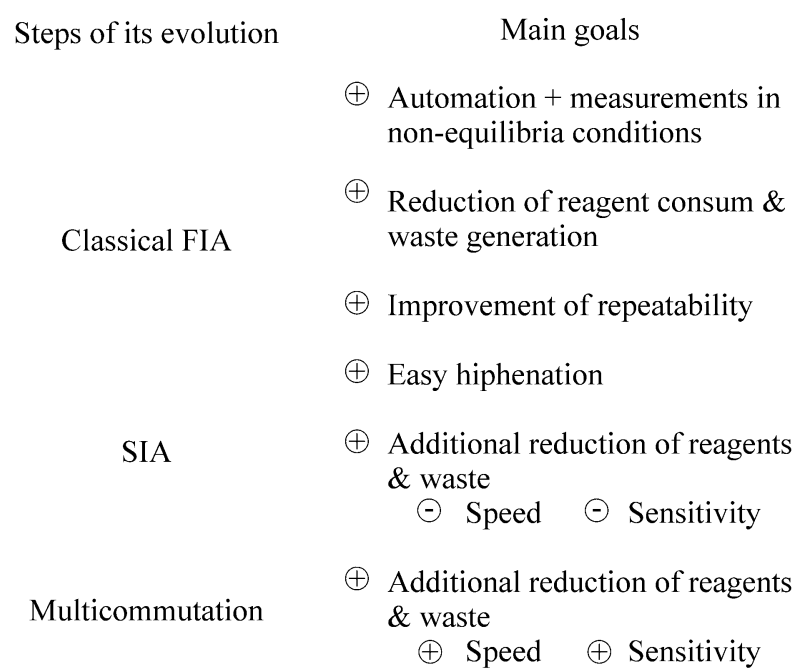

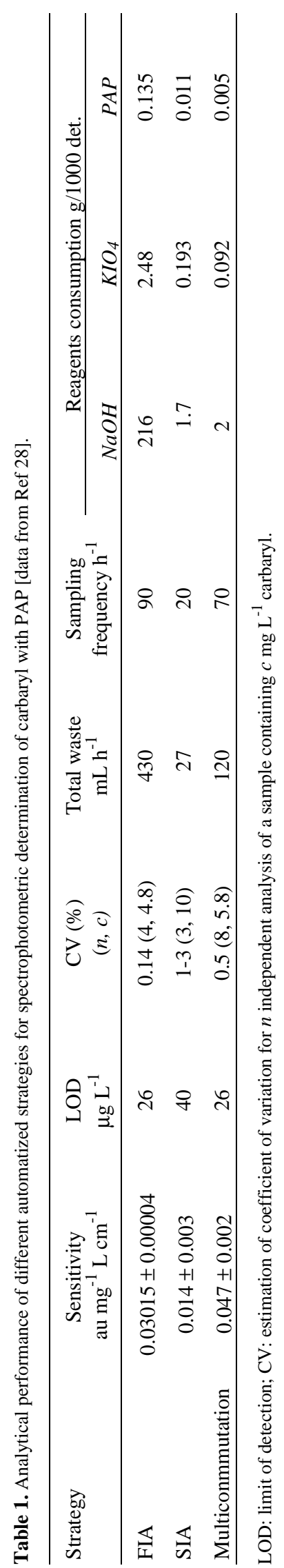

Batch $\longrightarrow$ FIA $\longrightarrow$ SIA $\longrightarrow$ Multicommutation

Figure 11. Evolution of flow analysis.

in 1957 on sequential injection analysis ${ }^{25}$, has provided a simple way to do that.

Figure 11 summarizes the main goals of the different flow analysis methodologies from 1975, paying a special attention to the simultaneous enhancement of laboratory productivity and reduction of wastes and reagent consumption, considering from classical FIA ${ }^{11}$ to sequential injection analysis (SIA) ${ }^{26}$ and multicommutation and binary sampling ${ }^{27}$. Table 1 compares the analytical performance of different automatized strategies for the spectrophotometric determination of carbaryl with p-aminophenol. From these data it can be concluded that multicommutation enhances the sensitivity of the classical FIA methods, reduces the wastes 25 or more times with a small reduction of the sampling rate and reduces the reagent consumption at levels comparable with those employed in SIA ${ }^{26}$.

\section{Conclusions}

Through the text, the most salient features of modern Analytical Chemistry have been presented, paying special attention to the integration of all these advances in measurement, sample and data treatment process in a global concept of the analytical process as a continuous operation to try to solve real problems and to provide valuable information from the chemical nature of materials.

In fact, the basic idea has been to provide an upgraded information about Analytical Chemistry, its fundamental principles and possibilities to all those who need accurate and precise data about their own systems but have not the specific formation in the field of Analytical Chemistry. 


\section{Acknowledgements}

The author acknowledges the financial support of the Spanish DGES Project PB96-0779.

\section{References}

1. Kellner, R.; Mermet, J.M.; Otto, M.; Widner H.M., eds, Analytical Chemistry, Wiley-VCH, Weinheim, 1998.

2. Malissa, H. Fresenius J. Anal. Chem. 1992, 343, 809.

3. Valcárcel, M.; Rios, A. Analyst 1995, 120, 2291.

4. Valcárcel, M.; Rios, A. Anal. Chem. 1993, 65, 781A.

5. Valcárcel, M.; Rios, A. Trends Anal. Chem. 1999, 18, 68.

6. Pardue, H.L. Chem. \& Eng. News 1983, 37, 38.

7. Pardue, H.L.; Woo, J. J. Chem.Educ. 1984, 61, 409.

8. Reis, B.F. Quim. Nova 1996, 19, 51.

9. Ruzicka, J.; Hansen, E.H. Flow Injection Analysis Wiley, New York, 1980.

10. Valcárcel, M.; Luque de Castro, M.D. Flow Injection Analysis: Principles and Applications Ellis Horwood, Chichester, 1987.

11. Ruzicka, J.; Hansen, E.H. Anal. Chim. Acta 1975, 78, 145.

12. de la Guardia, M. Mikrochim. Acta 1995, 120, 243.

13. Mason, T.J. Sonochemistry: The uses of ultrasound in Chemistry, RSC, Cambridge, 1990.
14. Morales-Rubio, A.; Pomares, F.; de la Guardia, M.; Salvador, A. J. Anal. At. Spectrom, 1989, 4, 329.

15. Pastor, A.; Vázquez, E.; Ciscar, R.; de la Guardia, M. Anal. Chim. Acta 1997, 344, 241.

16. Carbonell, V.; de la Guardia, M.; Salvador, A.; Burguera, J.L.; Burguera, M. Anal. Chim. Acta 1990, 238, 417.

17. de la Guardia, M.; Morales-Rubio, A. Trends Anal. Chem. 1996, 15, 311.

18. Burguera, M.; Burguera, J.L. Anal. Chim. Acta 1998, 366, 63.

19. Mermet, J.M. Analusis 1990, 18, 1.

20. Kowalski, B. J. Chem. Inf. Comput. Sci. 1975, 15, 201.

21. Massart, D.L.; Vandeginste, B.G.M.; Deming, S.N.; Michotte, Y.; Kaufman, L. Chemometrics: a textbook, Elsevier, Amsterdam, 1988.

22. de la Guardia, M.; Ruzicka, J. Analyst 1995, 120, 17N.

23. de la Guardia, M.; Khalaf, K.D.; Hasan, B.A.; Morales-Rubio, A.; Carbonell, V. Analyst 1995, 120, 231.

24. Sánchez-Dasí, J.; Garrigues, S.; Cervera, M.L.; de la Guardia, M. Anal. Chim. Acta 1998, 361, 253.

25. Skeggs, L.T. Am. J. Pathol. 1957, 28, 311.

26. Ruzicka, J.; Marshall, G.D. Anal. Chim. Acta 1990, 237, 329.

27. Reis, B.F.; Giné, M.F.; Zagatto, E.A.G.; Costa-Lima, J.L.F.; Lapa, R.A.S. Anal. Chim. Acta 1994, 293, 129.

28. Reis, B.F.; Morales-Rubio, A.; de la Guardia, M. Anal. Chim. Acta, in press. 